\title{
Semantic processing in dichotic listening? A replication*
}

\author{
ANNE TREISMAN, ROSEMARY SQUIRE, and JOANNE GREEN \\ Oxford University, Oxford, OX1 3PS, England
}

\begin{abstract}
This experiment explores semantic processing of one message while another is attended to and shadowed. It was an attempt to replicate and clarify an earlier finding by Lewis (1970). Like Lewis, we found that mean shadowing latency was increased when a synonym of the shadowed word coincided with it on the unattended channel. However, this occurred only with synonyms in an early list position and appeared to affect only a small proportion of trials. The implications of this and other recent findings for theories of selective listening are discussed.
\end{abstract}

Lewis (1970) reports a finding that has important implications for theories of selective attention to competing speech messages. He found that the shadowing latency to words on the attended ear increased when the coincident word on the unattended ear was a synonym and decreased when it was an associated word, although no words could be reported from the unattended ear. Lewis concludes that selection follows full perceptual and semantic analysis but precedes storage and admission to awareness. He justifiably criticizes most preceding experiments on selective listening, which claimed to test perception but either used a recall measure or required an immediate overt response which might conflict with a simultaneous response to the attended message. However, these criticisms do not apply to an experiment by Treisman and Riley (1969) which clearly showed restrictions on perceptual processing of simultaneous speech inputs when neither response conflict nor memory storage was involved. The very large increase that we found in the efficiency of detecting unattended target words when these differed in voice quality as well as semantic class cannot be explained by a model which assumes that full semantic analysis of all unattended words is possible. The results of these two experiments seem, at first sight, difficult to reconcile.

Two further papers have also reported experiments showing semantic processing of unattended dichotic messages and have argued against a perceptual interpretation of attention limits. Corteen and Wood (1972) found that GSRs were produced to $38 \%$ of shock-associated words and $23 \%$ of words from the same semantic class (names of cities) compared to control rates with neutral words of $12 \%$ and $9 \%$, i.e., increases of $26 \%$ and $14 \%$, respectively, due to the association with shock. Their Ss never subsequently reported any awareness of the city names, nor was there any increase in errors with the shadowing task. Mackay (1972) showed that a single word or pair of words on the unattended ear could bias the recognition of an

*The research was supported by a grant from the Medical Research Council. ambiguous sentence on the attended ear in the direction appropriate to their lexical meaning or to their surface structure, though not to their deep structure. For example, a sentence containing the word "bank" was more often interpreted in one way by Ss who simultaneously received the unattended word "river" and in another by Ss who received the word "money." Mackay took this as evidence for lexical and syntactic surface structure processing of unattended words. However, the effect was again a small one (ranging from $4 \%$ to $14 \%$ in the predicted direction), and the possibility of occasional switching of attention after the sentence on trials when the critical word occurred near the end was not precluded.

A more important criticism of both these papers is that neither gives any indication of the effect that would be obtained in conditions in which the shock-associated or biasing words were received on an attended channel and so were known to have been perceived. It seems quite possible that their effect would be more consistent than those obtained with unattended words. Yet a model that postulates full lexical analysis of unattended words should predict no difference in the number of GSRs or in the frequency of semantic biasing produced by attended and unattended words. In fact, the size of the effect in these two experiments is similar to or smaller than the proportion giving an overt response in Treisman and Riley's monitoring task. Thus, it would seem quite compatible with Treisman's suggestion that occasional semantically important or relevant words may be detected despite the reduction in $\mathrm{S} / \mathrm{N}$ ratio produced by filtering provided that conditions have induced Ss to lower the criteria within the speech recognition system for detecting them.

We are left with the question of what role awareness plays in selective listening. The results of these experiments, together with those of Treisman and Riley, suggest that subsequent verbal report is unlikely to occur unless detection of an unattended word causes Ss to stop shadowing and deliberately switch attention to the formerly unattended message. Our previous claim was not that identification of an unattended word inevitably 
brings with it a shift of attention, conscious awareness, and storage in memory, but only that it may do so. In an experiment in which contextually probable words from the unattended ear were occasionally shadowed (Treisman, 1960), Ss were, in fact, usually unaware that they had included a word from the wrong ear.

We return to Lewis's results, which pose more serious problems for the theory of perceptual limits in selective listening. It may be useful, however, to distinguish between two independent claims that Lewis could make: (1) that perceptual processing is complete for all inputs, i.e., that perceptual capacity is not limited; (2) that perceptual processing of simultaneous inputs is parallel rather than serial. The second could be true without entailing the first. Lewis's data do not and probably cannot prove the first claim. The missing control condition, as in Mackay's and Corteen and Wood's experiments, would be to measure the effect of the synonyms or associated words in conditions in which they were known to have been fully analyzed. Unfortunately, with Lewis's task there is no obvious way of doing this; this means equally that we have no way of knowing whether or how much the frequency of semantic processing of secondary words was reduced by concurrent processing of the primary words. Some indication might, however, be given by the variance and distribution of RTs. For example, if the meaning of unattended words is identified only on a proportion of the semantically related trials and never on control trials, the variance of shadowing latencies should be greater with the semantically related pairs. Lewis did not report the variance or distribution of individual RTs in his experiment; thus his published data are consistent with the hypothesis of either a small delay on all synonym pairs or a larger delay on a few synonym pairs.

The truth of the second claim, that perceptual processing of simultaneous inputs is parallel rather than serial, may vary with the nature of the task requirements. In focused attention tasks, filter theories claim that unattended messages are reduced in $\mathrm{S} / \mathrm{N}$ ratio. Processing with full attention is therefore necessarily serial, and switching channels involves resetting the filter. However, the modified filter theory (Treisman, 1964) also assumed some degree of parallel processing at lower levels of speech analysis, with filtered words dropping out before reaching full identification unless the S's criteria have been exceptionally lowered in their favor. In divided attention tasks, Ss would be less likely to adopt filtering as a strategy and more likely to attempt parallel processing. Two recent papers have reported evidence suggesting that perceptual capacity is limited, but that this capacity can be used in parallel if the task requires it. Treisman and Fearnley (1971) measured RTs to a single item or pair of items in a classification task. They found that advance knowledge of the identity of a target digit reduced the response latency equally when two simultaneous items were presented and when a single item was presented, suggesting parallel processing of the two items. However, responses to the pairs were considerably slower than responses to the single items, suggesting a limit to the capacity. Ninio and Kahneman ${ }^{1}$ measured detection rates and latencies when Ss listened to simultaneous lists of words for semantically defined target words. They found an increase from $7 \%$ to $26 \%$ in missed targets and an increase from 605 to $741 \mathrm{msec}$ in mean RT when Ss had to monitor two channels rather than one, but only a slight increase in the variance of RTs and no suggestion of bimodality. The results suggested parallel processing rather than alternating attention, but with a limited capacity shared between the two channels. Their results differ from those of Treisman and Davies (1973), whose Ss were unable to detect more than half the targets in an almost identical task. Thus, the extent to which parallel processing is possible depends on the degree to which the particular lists presented load the capacity of the particular Ss performing the task.

Lewis's experiment differs from these in requiring focused rather than divided attention. It would, therefore, be important for the theories to discover whether in his experiment those unattended words which were identified were processed in parallel with the attended words or serially. The latter, but not the former, would be compatible with the suggestion that unattended synonyms and associates are identified only because their meaning is related to that of the attended word and that other unattended words are not fully analyzed. Lewis's experimental paradigm seems well suited to answering this question: logically, the response to an attended word can be affected by the meaning of an unattended word only if the latter has previously been identified. Since $S$ s were asked to repeat each attended word as soon as they heard it, one could argue that identification of the two words must have occurred in parallel on those trials on which the unattended word affected the shadowing response. However, the shadowing latencies in Lewis's experiment were by no means the shortest possible, averaging about 675 msec. Marslen-Wilson (1973) has shown that accurate shadowing is possible for some Ss at lags of less than $300 \mathrm{msec}$. These Ss are more likely to differ from others in their motor efficiency than in their rate of perceptual processing, since speeded speech can generally be understood at rates of up to 5 or 6 words/sec. In Lewis's experiment, there should, therefore, at least occasionally have been time for serial identification of the unattended word before the shadowing response to the attended word began. This might be more likely to occur on trials on which the unattended synonym or associate started slightly ahead of the shadowed word. It would also be more likely to occur with Ss whose mean shadowing latency was long than with Ss who shadowed with very short latencies.

A final possibility is that it takes time for capacity to become fully loaded by the primary message and for attention to become efficiently focused on it. Thus, a 
Table 1

Mean Reaction Time, Square Root of Mean Variances, and Percent Errors for Each Type of Word Pair and Each Test Position

\begin{tabular}{lrrrr}
\hline & $\mathrm{S}_{3}$ & $\mathrm{C}_{3}$ & $\mathrm{~S}_{7}$ & $\mathrm{C}_{7}$ \\
\hline Mean RT & 565 & 532 & 617 & 633 \\
$\begin{array}{l}\text { Square root of mean variance } \\
\begin{array}{l}\text { Percent errors, omissions, or } \\
\text { intrusions on critical word or } \\
\text { following word }\end{array}\end{array}$ & 129 & 92 & 115 & 126 \\
\hline
\end{tabular}

change from some degree of parallel processing to more exclusively single-channel processing may become increasingly necessary and likely as the two messages continue. Lewis did not report the effect of serial position in his first experiment, in which he presented test pairs of the same type in the third, fifth, seventh, and ninth positions of 11 -word lists. In his second experiment, he used lists of only 5 words in the primary message with just one secondary word coinciding with the third word in the list.

We decided to replicate Lewis's experiment with slight modifications in order to throw more light on the nature of the interference caused by synonym pairs, its effect on the distributions of RTs, and its variation with serial position. Only one synonym pair was given in each list, and its position was always either third or seventh in a list of 12 pairs. Each shadowed word was paired with a word on the other ear to ensure that capacity would be as fully loaded as in the usual selective listening situation. This contrasts with Lewis's second experiment, in which only the third (critical) word was paired with one on the other ear.

\section{METHOD}

\section{Experimental Material}

Twenty-four lists of 10 pairs of words were made up by random selection from all monosyllables in the 1,000 highest frequency words in Thorndike-Lorge's word frequency count. Forty-eight pairs of synonyms were also collected from the same source, except that 11 of the 96 words had to be selected from an increased pool of words in order to get 48 pairs of monosyllables that were sufficiently similar in meaning. The lowest frequency used was 20 per million. The synonyms were not identical in meaning (it is unlikely that any such pairs of words exist), but they were closely similar, e.g., hurt-harm, big-large, shape-form. Two critical pairs of words were added to each list of 10 neutral pairs -a synonym pair in Position 3 or 7 and a control pair in the other of these two positions. Half the lists had the synonyms in Position 3 and the control pairs in Position 7 and half had the reverse arrangement. The control pairs consisted of the same words as the synonym pairs, but randomly repaired to ensure that the two words in each pair were unrelated in meaning. Each list was prefaced by the words "one, two, three, four" spoken at the same rate as the list pairs.

Two separate recordings were made of these 24 lists of 12 pairs of words. The top tracks of the two recordings were identical, while the bottom tracks differed only in that the word which, in one case, had been paired with a synonym was in the other case paired with a control word, and vice versa. One group of six Ss was tested on one recording and another on the other. The recordings were made by a female speaker at a rate of 3 pairs every $2 \mathrm{sec}$. The recordings were checked by means of an oscillograph made on a UV recorder to measure the degree of asynchrony between the words in the critical pairs. The mean asynchrony irrespective of direction was $40 \mathrm{msec}$, and the range was -112 to $+143 \mathrm{msec}$. There was no consistent relation between the type of pair and the degree or the direction of asynchrony. Twelve practice lists were also recorded without synonym pairs and consisting of slightly lower frequency monosyllabic words (Thorndike-Lorge frequency between 10 and 50 per million).

\section{Apparatus}

The lists were presented on a Revox two-channel stereo tape recorder over a pair of padded Sony TC 150 earphones. As the S listened and shadowed, the track he was shadowing was re-recorded onto another Revox tape recorder; simultaneously his shadowing responses were recorded on the other track. Oscillograph recordings were later made of the critical words, together with the $\mathrm{Ss}$ ' shadowing responses to them, and the interval between the onset of the critical words and the onset of the responses was measured. Accuracy was to the nearest $5 \mathrm{msec}$.

\section{Procedure}

Ss were given at least 24 practice trials at shadowing, using each track of the practice lists and reusing them if more than 24 trials were needed to reach a criterion of 5 consecutive lists averaging, at most, 1.0 shadowing error per list (however minor, e.g., a single phoneme altered). (A number of Ss were discarded because they failed to reach this criterion in 48 practice trials.) They were then given the experimental lists to shadow and told to ignore the words in the nonshadowed ear. Half the Ss in each group shadowed the list in their right ear and half the list in their left ear. They were asked to repeat each word as soon as they heard it and, if they should miss any, to repeat back the current word rather than lagging behind to make good the omissions.

\section{Scoring}

The shadowing responses were categorized as follows: correct, omission, error in one or more phonemes, intrusion of one or more phonemes from the wrong ear. No intrusions of complete words occurred. Response times for the critical words were measured only if the critical word was shadowed correctly, and no omission, intrusion, or error was made on the two preceding words or the one succeeding word. The minimum number of response times remaining for any $\mathrm{S}$ in any category $\left(\mathrm{S}_{3}=\right.$ synonym in Position $3, S_{7}=$ synonym in Position $7, C_{3}=$ control word in Position 3, $\mathrm{C}_{7}=$ control word in Position 7) was 5 , and the mean was 8.1 out of 12 .

\section{RESULTS}

Table 1 shows the means of the RTs in each condition, the square root of the mean of the individual variances, and the percent errors or omissions on the critical word and the one following it. Analysis of variance showed no significant main effect of synonym vs control words, no effect of lists (Set A vs Set B), a significant effect of list position $[F(1,10)=26.0$, $\mathrm{p}<01]$, indicating that responses were slower in Position 7 than in Position 3, and a significant interaction between synonym vs control words and list position $([F(1,10)=7.6, p<.05]$. A test on the simple main effects showed that the difference between synonym and control pairs was significant at Position 3 $[F(1,20)=6.5, p<.05]$, but not at Position 7 (where it went, if anything, in the reverse direction). An analysis of variance on the errors to the critical word and the one 

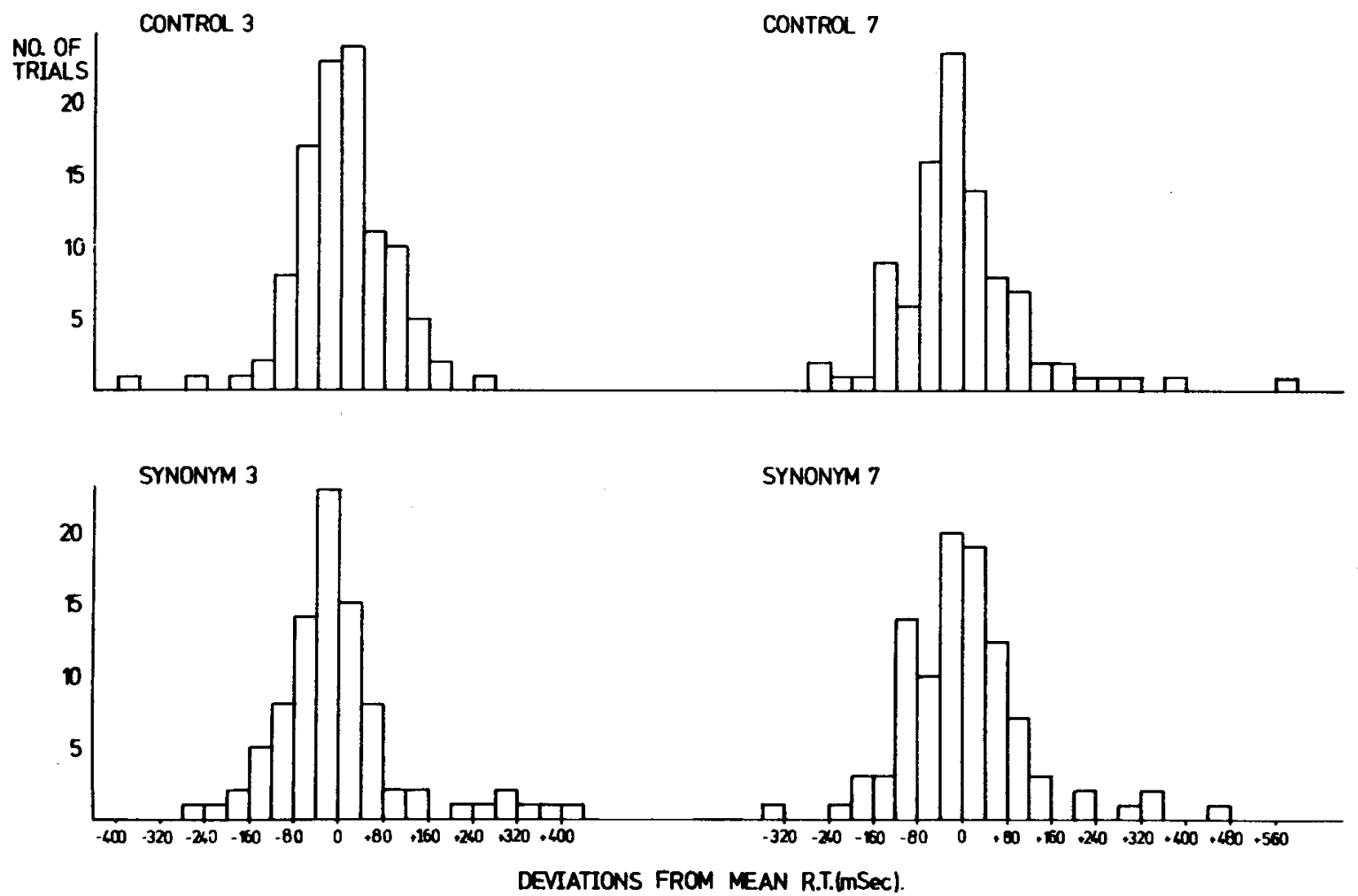

Fig. 1. Pooled distributions of latencies, shown as deviations from each S's mean latency to each type of word pair.

following it showed that the slightly greater number of errors produced with the synonyms did not differ significantly from that produced by the control words.

An analysis of the individual variances showed a significant effect of list position $[F(1,10)=5.64$, $\mathrm{p}<.05]$, but the difference between synonym and control pairs did not reach significance and neither did the interaction. The pooled distributions for all Ss are shown in Fig. 1 as deviations from each S's mean response times to each type of word pair. Although the variances did not differ significantly, it is worth noting that if the longest RT is dropped from each $S$ in the $S_{3}$ and in the $C_{3}$ condition, the difference between the means is reduced from 33 to $15 \mathrm{msec}$ and is no longer significant on $t$ test $[t(11)=1.6]$. This suggests that the difference between the means at Position 3, which did occur consistently across Ss (shown by 11 out of $12 \mathrm{Ss}$ ), was due largely to one or two very long RTs on the synonym pairs rather than to a small increase in RT on every trial.

The fact that control words in Position 7 actually gave slightly longer latencies than synonym words was surprising. One possible explanation is that the interference produced by the synonyms in Position 3 actually extended far beyond the response to the synonyms themselves and produced a delay on all subsequent words at least up to the seventh. Since the control words in Position 7 always followed a synonym pair in Position 3, this prolonged interference might have been greater than any immediate interference produced by synonyms in Position 7, and so have resulted in the unexpected reversal in the RTs. Lewis's lists in the first experiment always contained the same type of pairs in all critical positions. Thus, synonyms in the early list positions might have produced a cumulative delay. This could explain why the mean effect was greater in Lewis's first experiment than in his second experiment, in which only one synonym pair occurred in each list.

We therefore decided to measure shadowing latencies for words in other list positions, in order to see whether this hypothesis of continued interference was correct. Unfortunately, by this time the recordings of 5 of the 12 Ss had been erased, so this analysis was possible only for 7 Ss. Figure 2 shows the mean shadowing latencies for these $7 \mathrm{Ss}$ for the two types of lists, and the mean number of RTs per $S$ which contributed to each point. The data for synonym pairs are indicated by circles. Clearly the delay caused by the synonym in Position 3 persisted almost to the end of the list. There is no evidence of a change in relative shadowing latencies for the two lists at Positions 7,8, or 9, and an analysis of variance on the words from Position 4 to the end showed no interaction $(\mathrm{F}<1)$ between list position and list type $\left(\mathrm{S}_{3} \mathrm{C}_{7}\right.$ vs $\left.\mathrm{C}_{3} \mathrm{~S}_{7}\right)$; this should have been present if a new source of interference were introduced by the synonym pair in Position 7. The difference in latencies disappears for the last two words; however, performance on these was probably affected by Ss' knowledge that the list was ending; both the mean and the variance of latencies increased and the mean number of measurable latencies dropped rather low.

We wished to see whether the delays caused by 
Fig. 2. Mean shadowing latencies for seven Ss, and mean number of latencies measured per $S$.
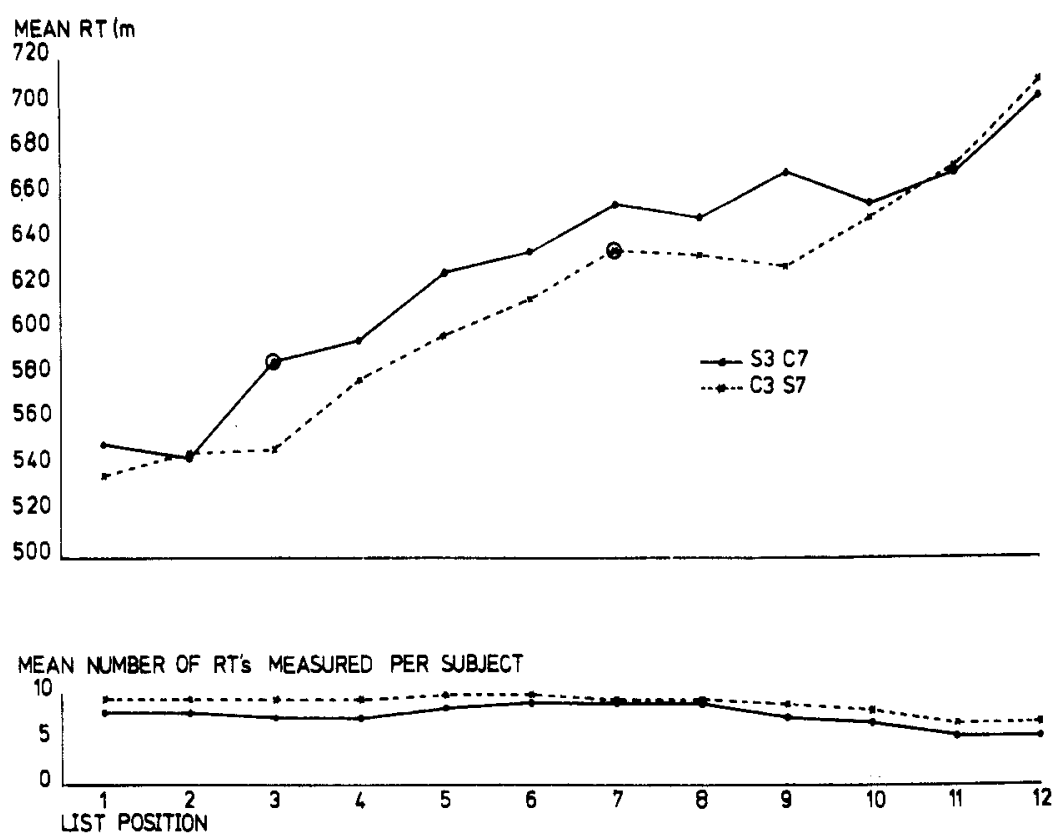

synonyms were more likely to occur on those trials on which the synonym happened to start earlier than the shadowed word. We therefore rank-ordered each S's RTs to critical pairs according to the extent to which the unattended word's onset preceded that of the attended word. The mean RTs for those above the median were $580 \mathrm{msec}$ for $\mathrm{S}_{3}$ and $534 \mathrm{msec}$ for $\mathrm{C}_{3}$ pairs, while for those below the median they were 550 and $532 \mathrm{msec}$. Although these means appear to support the hypothesis, neither the effect of the asynchrony nor its interaction with $\mathrm{S}_{3}$ vs $\mathrm{C}_{3}$ reached significance on analysis of variance. However, 10 out of $12 \mathrm{Ss}$ showed a greater effect of the direction of asynchrony with $\mathrm{S}_{3}$ than with $\mathrm{C}_{3}$ pairs, which has a chance probability of only 0.019 (by the sign test).

Finally we tested the idea, arising from the serial processing model, that delays should occur chiefly for Ss whose normal shadowing latency was long. The range of Ss' mean latencies to $\mathrm{C}_{3}$ words was 317 to $730 \mathrm{msec}$ with a mean of 532 . The correlation between mean $\mathrm{C}_{3}$ latencies and the difference between $S_{3}$ and $C_{3}$ latencies was positive but not statistically significant $[\mathrm{r}(10)=$ $0.32]$. It could be that long latencies are necessary but not sufficient to allow identification of secondary words. The two $\mathrm{Ss}$ with mean $\mathrm{C}_{3}$ latencies under $350 \mathrm{msec}$ showed no delay with $S_{3}$ pairs at all (a mean of $-5 \mathrm{msec}$ ), while the mean latency to $\mathrm{C}_{3}$ pairs of the six Ss showing the biggest delay (a mean of $61 \mathrm{msec}$ ) was $575 \mathrm{msec}$.

\section{DISCUSSION}

This experiment confirms Lewis's finding, but also clarifies its nature and defines further the conditions under which it can be obtained. Although our Ss tended to shadow with a shorter lag than his, the increase in latency comparing synonyms to unrelated words was of the same order for our $S_{3}$ words and his second experiment ( $33 \mathrm{msec}$ compared to $39 \mathrm{msec}$ ). Our results show equally clearly that the semantic interference arises only with pairs occurring early in the list. By the seventh pair (or earlier), the meaning of the secondary words has stopped having any effect on shadowing latencies. The results are therefore consistent with the suggestion that it takes a short time for capacity to become fully occupied by one channel and for the other to be effectively excluded from semantic analysis. They give no support to the claim that the meaning of secondary words is fully analyzed at all test positions, although, admittedly, they do not exclude the possibility either. One could argue that the change with list position is a change in the degree to which the shadowing response is vulnerable to interference rather than a change in the source of interference itself. Another possibility is that the continued delays on the $S_{3} / C_{7}$ lists are due to an increased tendency to keep sampling the unattended channel, resulting from the detection of a relevant word there (the synonym). Thus the degree to which the available capacity is focused on the primary channel may affect the latency of responses to it. This possibility could be tested by comparing detection probabilities for target words in the unattended channel in lists in which they follow synonym pairs as opposed to neutral words.

The questions of whether all or only a small proportion of synonyms in early list positions delay RTs to the words they are paired with and whether their analysis is serial or parallel are more difficult to answer. The variances of individuals' RTs were large and differed considerably across Ss, so that analysis of variance showed no significant difference between $\mathrm{S}$ and $\mathrm{C}$ variances. However, both the mean variances and the distributions would be consistent with the hypothesis 
that the delays occurred with only a small proportion of synonym pairs. The tendency for the long delays to occur chiefly on trials on which the onset of the synonym happened to precede the onset of the shadowed word is also consistent with this interpretation, although again not conclusive. Early identification could increase the opportunity for interference relative to late identification (Lewis's hypothesis) as well as to nonidentification.

The delays did not occur for the two Ss who shadowed with very short lags, although for Ss with lags of $400 \mathrm{msec}$ or more there was little correlation between mean delay and mean lag. This result does not preclude serial processing but leaves its occurrence uncertain, and therefore also leaves open the question of whether synonyms were more likely to be identified than control words just because they were synonyms.

This experiment again illustrates the difficulty of finding conclusive evidence for (or indeed against) consistent and unimpaired semantic analysis of "unattended" words. This is not, of course, an argument against its existence. However, in the light of the drastic reduction in responses to unattended semantic targets shown in Treisman and Riley's experiment (1969), which excluded both memory and response as sources of the difficulty, the onus appears to be on those who hold the more extreme hypothesis that all inputs reach full semantic identification. It might be tempting but not, we think, justifiable to conclude that no empirical test of the models can be made. One possibility would be to require a response which was conditional on the meaning of both words in a pair. If simultaneous words (presented in rapid sequences) can be identified only when they are related in meaning, the task should be possible only with related words and never with unrelated words. The proportion of trials on which Ss were successful might then indicate whether the analysis of related words occurs consistently or only on a small proportion of trials.

\section{REFERENCES}

Corteen, R. S., \& Wood, B. Autonomic responses to shock-associated words in an unattended channel. Journal of Experimental Psychology, 1972, 94, 308-313.

Lewis, J. L. Semantic processing of unattended messages using dichotic listening. Journal of Experimental Psychology, 1970 , 85, 225-228.

Mackay, D. G. Aspects of the theory of comprehension, memory and attention. Quarterly Journal of Experimental Psychology, $1973,25,22-40$.

Marslen-Wilson, W. Linguistic structure and speech-shadowing at very short latencies. Nature, 1973, 244, 522-523.

Treisman, A. M. Contextual cues in selective listening. Quarterly Journal of Experimental Psy chology, 1960, 12, 242-248.

Treisman, A. M. Selective attention in man. British Medical Bulletin, 1964, 20, 12-16.

Treisman, A. M., \& Davies, A. Divided attention to ear and eye. In S. Kornblum (Ed.), Attention and performance IV. New York and London: Academic Press, 1973. Pp. 101-117.

Treisman, A. M., \& F earnley, S. Can simultaneous speech stimuli be classified in parallel? Perception \& Psychophysics, 1971, 10. 1-7.

Treisman, A. M., \& Riley, J. G. A. Is selective attention selective perception or selective response? A further test. Journal of Experimental Psychology, 1969, 79, 27-34.

\section{NOTE}

1. Personal communication concerning "Reaction time in focused and in divided attention," a manuscript in preparation.

(Received for publication February 1, 1974; accepted March 18, 1974.) 